\title{
Redução de Danos: estratégia de cuidado com populações vulneráveis na cidade de Santo André - SP
}

\section{Harm Reduction: strategic care for vulnerable populations in the city of Santo André - SP}

\author{
Silvia Moreira da Silva \\ Assistente Social. Coordenadora da Unidade de Redução de Danos \\ - Programa de Saúde Mental da Secretaria Municipal de Saúde \\ de Santo André. Mestre em Enfermagem de Saúde Coletiva pela \\ Faculdade de Enfermagem da Universidade de São Paulo. \\ Ana Lucia Spiassi \\ Socióloga. Coordenadora do Núcleo de Prevenção em DST/AIDS da \\ Secretaria Municipal da Saúde de Santo André. Pesquisadora do \\ Centro de Estudos em Saúde Coletiva da Faculdade de Medicina \\ do $A B C$ e mestranda em Medicina Preventiva pela Faculdade de \\ Medicina da Universidade de São Paulo. \\ Endereço: Rua das Silveiras, 73, Vila Guiomar, CEP 0907ı-100, \\ Santo André, SP, Brasil. \\ E-mail: spiassiðuol.com.br

\section{Decio de Castro Alves} \\ Psicólogo. Coordenador do Programa de Saúde Mental da Secre- \\ taria de Saúde de Santo André.

\section{Daniela de Jesus Guedes} \\ Licenciada em computação. Redutora de Danos da Unidade de \\ Redução de Danos da Secretaria Municipal de Saúde de Santo \\ André.

\section{Reinaldo de Oliveira Leigo} \\ Técnico em Enfermagem. Redutor de Danos da Secretaria Municipal \\ de Saúde de Santo André.
}

\section{Resumo}

A Unidade de Redução de Danos (URD) da Secretaria Municipal de Saúde de Santo André, compreendida como instrumento do serviço de saúde em atuação avançada, tem por finalidade transformar a situação de saúde de sujeitos que fazem parte de grupos sociais estigmatizados e, portanto, vulnerabilizados; são indivíduos que circulam ou trabalham nas ruas - usuários de drogas, michês, mulheres profissionais do sexo, homens que fazem sexo com homens, adolescentes em situação de exploração sexual, transexuais, travestis, lésbicas e mulheres que trabalham em casas de programas. Nosso objetivo é assegurar aos indivíduos desses grupos o direito à saúde e, baseados no princípio de Integralidade do SUS, apoiar o acesso a outros direitos sociais. Desde 2002, através do trabalho de campo, foi possibilitada a vinculação de 240 profissionais do sexo, 120 travestis, 10 crianças e adolescentes, 28 usuários de droga injetável e usuários de crack, que até então não tinham acesso aos recursos e dispositivos de saúde do Município.

Palavras-chave: Redução do dano; Drogas de abuso; Avaliação de serviços de saúde; Problemas sociais. 


\section{Abstract}

The Harm Reduction Unit (HRU) of the Municipal Health Department of Santo André (state of São Paulo) is a working tool for healthcare that has the purpose of transforming the health situation of individuals who are part of socially stigmatized, therefore vulnerable, groups. These are individuals who spend their lives or work on the streets: drug users, male prostitutes, female sex workers, men who have sex with men, sexually exploited adolescents, transsexuals, transvestites, lesbians and women working in brothels. The HRU's potential benefits were indicated to be: it improved healthcare and condom use; decreased drug use; improved seeking and access to healthcare services; decreased material sharing for drug use; improved decision-making capacity. HRU's actions have gone beyond prevention of health damage, as it has promoted broader transformations such as awareness of social rights in general and health rights in particular, along with actions towards making these rights apply, based on the principle of integrality of SUS (Brazil's National Health System). Since 2002 we have assisted, through outreach work, 240 prostitutes, 120 travestites, 10 sexually exploited adolescents and 28 drug users (intravenous and crack).

Keywords: Harm Reduction; Street Drugs; Health Service Evaluation; Social Problems.

\section{Caracterização do Problema}

A Unidade de Redução de Danos (URD) da Secretaria Municipal de Saúde de Santo André, compreendida como instrumento do serviço de saúde em atuação avançada, tem por finalidade transformar a situação de saúde de sujeitos que fazem parte de grupos sociais estigmatizados e, portanto, vulnerabilizados; são indivíduos que circulam ou trabalham nas ruas - usuários de drogas, michês, mulheres profissionais do sexo, homens que fazem sexo com homens, adolescentes em situação de exploração sexual, transexuais, travestis, lésbicas e mulheres que trabalham em casas de programas.

Nosso objetivo é assegurar aos indivíduos desses grupos o direito à saúde e, baseados no princípio de Integralidade do SUS, apoiar o acesso a outros direitos sociais.

Para a população adstrita ao programa trabalhar e viver nas ruas ou em locais de moradia inadequados são consequências de uma trajetória de exclusão dos sistemas sociais amplos e da impossibilidade de acessar outros tipos de inserção na cidade.

\section{Descrição}

A partir de junho de 2002, o município de Santo André organizou suas primeiras ações de redução de danos, tendo como público principal as crianças e adolescentes em situação de exploração sexual que, na sua grande maioria, o fazem em troca de facilidades para se conseguir cocaína e crack. Essas ações visavam obter um melhor diagnóstico da situação no Município, tendo como metodologia o trabalho de campo realizado por redutores de danos que, no contato com esse público, buscava orientá-los em relação à prática de sexo seguro, cuidados básicos com a saúde, vinculação a projetos socioeducativos e de reinserção familiar etc.

Em abril de 2003, as ações realizadas de maneira integrada entre o Programa de Saúde Mental e DST/AIDS foram ampliadas para um público mais abrangente, envolvendo profissionais do sexo, travestis, usuários de droga injetável e outras drogas. Dentre as ações realizadas se destacam as de prevenção em relação às doenças transmissíveis, redução de riscos e danos relacionados ao consumo de substâncias psicoativas, de incentivo ao protagonismo e organização dos usuários, 
vinculação à rede de saúde e incorporação de usuários ao trabalho de redução de danos.

A estratégia de acesso e oferta de insumos e serviços do modelo da redução de danos é o momento da busca do conhecimento e do contato com a realidade dos sujeitos a partir de suas escolhas e possibilidades concretas de mudanças. Ou seja, ter como meta a redução de riscos e danos associados a comportamentos tidos como potencialmente prejudiciais ao indivíduo traz, a nosso ver, maiores possibilidades de resultar em pequenas mudanças no estilo de vida sem demandar alterações radicais das práticas e escolhas pessoais dos usuários.

O trabalho de campo possibilitou a vinculação de 240 profissionais do sexo, 120 travestis, 10 crianças e adolescentes, 28 usuários de droga injetável e usuários de crack, que até então não tinham acesso aos recursos e dispositivos de saúde do Município.

\section{Metodologia de Trabalho}

1. Mapeamento de regiões frequentadas pela população abrangida pela proposta;

2. Definição de locais, dias e horários que atuarão como pontos de referência da Unidade Móvel para a população adstrita;

3. Oferta de serviços nos locais definidos: diagnóstico sorológico da infecção pelo HIV; coleta de sangue, Papanicolau e escarro; vacinas contra Hepatite B, Dupla Adulto e Tríplice Viral; clínica de abordagem sindrômica de DSTs; orientação e aconselhamento em relação à prevenção e ao tratamento de doenças e diminuição de riscos e danos relacionados ao consumo de substâncias psicotrópicas; fornecimento de insumos para prevenção e redução de danos (preservativos masculino e feminino, luva, lubrificante, kits de redução de danos, material informativo);

4. Encaminhamentos para inclusão em programas da rede de saúde, tratamento para dependência de álcool e outras drogas, dispositivos sociais e de inserção no trabalho.

\section{Lições Aprendidas}

Ao avaliar nossa prática procuramos utilizar a informação produzida para demonstrar a validade dos pressupostos inicialmente levantados (Novaes, 200o).
Em recente trabalho desenvolvido dentro do programa de pós-graduação do departamento de Enfermagem em Saúde Coletiva da USP, Silva (2008) trata da avaliação das ações da Unidade de RD de Santo André, ocasião em que pudemos refletir que, sobre a relação dos profissionais de saúde, da própria URD e da rede como um todo, é preciso que o saber dos usuários seja ouvido e valorizado (Ayres, 2001).

Com a produção de serviços em larga escala e passando os trabalhos a serem organizados segundo as complexas estruturas das instituições, este fato tornou inevitável uma certa despersonalização nos cuidados prestados (Schraiber e Mendes-Gonçalves, 1996, p. 38).

Brites (1999) assinala a importância do desenvolvimento de ações de redução de danos em situações concretas, muito diferentes do que pode ser percebido como ideal pelos serviços de saúde. Muitas vezes, a população acessada pela URD encontra-se cansada das imposições institucionais e sociais que não têm a menor condição de serem cumpridas, como, por exemplo, a exigência de abstinência como condição para o tratamento. Tais imposições têm feito com que usuários que necessitam de atenção de saúde fiquem sem assistência: os serviços de saúde não consideram aceitáveis alternativas à abstinência, como diminuir o consumo de algumas drogas ou eventualmente trocar por outras consideradas menos problemáticas.

\section{Recomendações}

Pode-se concluir que a URD está sendo instrumental para que o grupo social a que se dirige possa acessar direitos sociais, especialmente o direito à saúde. Vários são os testemunhos que relatam nesse sentido: os usuários referem-se à redução de danos como um facilitador, um intermediário entre as demandas que apresentam e os serviços sociais e de saúde; posicionando-se como cidadãos, não se comportam como subalternos junto aos serviços para os quais são encaminhados pela redução de danos; tornaram-se também agentes dos direitos sociais, pois apresentam aos demais as informações que têm obtido, levando outros usuários para serem atendidos nos locais em que já foram assistidos; avaliaram criticamente a URD, formulando sugestões para o seu aprimoramento, demonstrando conhecimento e familiaridade com a Unidade e mostrando-se à vontade 
para tecerem críticas e sugestões, sem receio de serem alvos de retaliações no atendimento; ficou evidenciado que os usuários têm vínculo e confiança na URD, verbalizando desejos e solicitações e contribuindo para a construção desse serviço de saúde.

Na avaliação dos usuários, a URD é um serviço cuja continuidade deve ser garantida pelo poder público, pois possui uma proposta positiva de acolhimento, acompanhamento e encaminhamento, que respeita os direitos humanos de um grupo social vulnerabilizado (Silva, 2008) e, em nosso entendimento, poderia ser adotado por outras cidades e melhor apoiado pelas instâncias de financiamento do SUS.

Este projeto é um esforço para consolidar as diretrizes do Sistema Único de Saúde, como acessibilidade, integralidade, equidade e aproximação dos usuários aos serviços de saúde e da rede socioassistencial. Com essa experiência de implantação das estratégias da redução de danos, tem sido possível alcançar populações vulneráveis e, com a oferta de cuidados, contribuir para a aproximação desses usuários aos seus direitos como cidadãos.

\section{Referências}

AYRES, J. R. C. M. Sujeito, intersubjetividade e práticas de saúde. Ciência e Saúde Coletiva, Rio de Janeiro, v. 6, n. 1, p. 63-72, 2001.

BRITES, C. M. Uso de droga injetável e redes de interação social: prazer e cuidados com a saúde, é possível? 1999. Dissertação (Mestrado em Serviço Social).São Paulo: Pontifícia Universidade Católica de São Paulo, 1999.

SCHRAIBER, L. B.; MENDES-GONÇALVES, R. B. Saúde do adulto: programas e ações na unidade básica. São Paulo: Hucitec, 1996.

NOVAES, H. M. D. Avaliação de programas, serviços e tecnologias em saúde. Revista de Saúde Pública, São Paulo, v. 34, n. 5, p. 547-59, 2000.

SILVA, S. M. As práticas de atenção à saúde da Unidade de Redução de Danos do município de Santo André: uma avaliação. 2008. Dissertação. São Paulo: Escola de Enfermagem da Universidade de São Paulo, 2008. 